\title{
Regenerating time series from ordinal networks
}

Michael McCullough, Konstantinos Sakellariou, Thomas Stemler, and Michael Small

Citation: Chaos 27, 035814 (2017); doi: 10.1063/1.4978743

View online: https://doi.org/10.1063/1.4978743

View Table of Contents: http://aip.scitation.org/toc/cha/27/3

Published by the American Institute of Physics

\section{Articles you may be interested in}

Small-world bias of correlation networks: From brain to climate

Chaos: An Interdisciplinary Journal of Nonlinear Science 27, 035812 (2017); 10.1063/1.4977951

Introduction to Focus Issue: Complex network perspectives on flow systems

Chaos: An Interdisciplinary Journal of Nonlinear Science 27, 035601 (2017); 10.1063/1.4979129

A complex network representation of wind flows

Chaos: An Interdisciplinary Journal of Nonlinear Science 27, 035808 (2017); 10.1063/1.4977699

Climate network stability measures of El Niño variability

Chaos: An Interdisciplinary Journal of Nonlinear Science 27, 035801 (2017); 10.1063/1.4971784

Inferring directed climatic interactions with renormalized partial directed coherence and directed partial correlation

Chaos: An Interdisciplinary Journal of Nonlinear Science 27, 035815 (2017); 10.1063/1.4978548

Percolation framework to describe El Niño conditions

Chaos: An Interdisciplinary Journal of Nonlinear Science 27, 035807 (2017); 10.1063/1.4975766 


\title{
Regenerating time series from ordinal networks
}

\author{
Michael McCullough, ${ }^{1, a), b)}$ Konstantinos Sakellariou, ${ }^{1, b}{ }^{1,}$ Thomas Stemler, ${ }^{1,2}$ \\ and Michael Small ${ }^{1,2,3}$ \\ ${ }^{1}$ School of Mathematics and Statistics, The University of Western Australia, Crawley, \\ Western Australia 6009, Australia \\ ${ }^{2}$ Complex Data Modelling Group, Faculty of Engineering, Computing and Mathematics, \\ The University of Western Australia, Crawley, Western Australia 6009, Australia \\ ${ }^{3}$ Mineral Resources, CSIRO, Kensington, Western Australia 6151, Australia
}

(Received 7 November 2016; accepted 6 March 2017; published online 22 March 2017)

\begin{abstract}
Recently proposed ordinal networks not only afford novel methods of nonlinear time series analysis but also constitute stochastic approximations of the deterministic flow time series from which the network models are constructed. In this paper, we construct ordinal networks from discrete sampled continuous chaotic time series and then regenerate new time series by taking random walks on the ordinal network. We then investigate the extent to which the dynamics of the original time series are encoded in the ordinal networks and retained through the process of regenerating new time series by using several distinct quantitative approaches. First, we use recurrence quantification analysis on traditional recurrence plots and order recurrence plots to compare the temporal structure of the original time series with random walk surrogate time series. Second, we estimate the largest Lyapunov exponent from the original time series and investigate the extent to which this invariant measure can be estimated from the surrogate time series. Finally, estimates of correlation dimension are computed to compare the topological properties of the original and surrogate time series dynamics. Our findings show that ordinal networks constructed from univariate time series data constitute stochastic models which approximate important dynamical properties of the original systems. Published by AIP Publishing.
\end{abstract}

[http://dx.doi.org/10.1063/1.4978743]

It can be useful to build models based solely on observed time series data when attempting to analyse complex nonlinear systems for which the driving mechanism is not fully understood. For example, an ordinal network is such a model produced by mapping time series to a set of symbolic states based on order patterns in short segments of data and then constructing an abstract network representation of the time series based on the temporal succession of these states. Thus far, this class of models has primarily been investigated as a tool for the computation of measures that are sensitive to the properties of the dynamics. However, if ordinal networks are actually encoding the information necessary to capture the essence of complex dynamics, then it should be possible to generate new time series from these models which have similar properties to the original data from which they are built. Herein, we investigate this hypothesis.

\section{INTRODUCTION}

The concept of transforming or mapping time series data to a network was first proposed ten years ago by Zhang and Small as a means of gaining additional insight into data from complex systems. ${ }^{1}$ The fundamental premise is that once data have been mapped to a network, various measures from the field of network science can be applied to extract

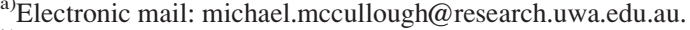

${ }^{\mathrm{b})} \mathrm{M}$. McCullough and K. Sakellariou contributed equally to this work.
}

quantitative measures that relate back to the dynamics of the system from which the time series was measured. There now exist numerous methods for network based time series analysis and some, such as recurrence networks, have been developed into a well established framework. ${ }^{2}$ To investigate how much information is encoded in a network model of a time series, some studies have been undertaken to recover the original time series from the network, to use the network to reconstruct the phase space topology of the original system, or to generate new time series from the networks and compare these with the original. ${ }^{3-7}$

In this paper, we apply this type of approach to the study of a special class of network based time series models called ordinal networks. ${ }^{8}$ These are transition networks constructed based on the temporal succession of states defined by the ordinal symbolic dynamics of a time series. ${ }^{9}{ }^{910}$ Simple measures on ordinal networks such as mean node degree and network diameter have been shown to be effective for change point detection. ${ }^{8}$ Furthermore, the partition that defines the ordinal symbolic dynamics is of particular interest because it has the generating property under specific conditions. ${ }^{11,12}$

The aim of this research is to investigate the extent to which ordinal networks encode the dynamics of discrete sampled time series from continuous chaotic systems in the structure of the networks. To do this, we take random walks on the network to regenerate new symbolic dynamics and use a data reassignment procedure to produce surrogate time series. Then, we compute invariant measures and recurrence properties to compare the original time series with the 
regenerated surrogate. The remainder of the paper is structured as follows: In Section II, we define the method for constructing ordinal networks from time series and the random walk algorithm and data reassignment procedure for regenerating surrogates. The main investigation is divided into three main sections. First, in Section III, we present a qualitative analysis of the random walk surrogate time series. Second, in Section IV, we use order recurrence plots, ${ }^{13}$ recurrence quantification analysis, ${ }^{14}$ and estimates of the largest Lyapunov exponent to study the case for ordinal networks constructed with a time lag and random walks which are constrained by incorporating a memory of previous states. Third, in Section V, we investigate the case of ordinal networks that are defined without a time lag and Markovian random walks for the generation of surrogates. In this section, we utilise the aforementioned methods of analysis as well as the time delayed mutual information and correlation dimension. Our findings are summarised in Section VI.

Note that in the main body of this paper, we elected to only show results for time series generated by the Lorenz system $^{15}$ for the sake of brevity and comparability of results. A brief report comprising selected results for other well known chaotic systems is included as supplementary online material.

\section{METHODOLOGY}

\section{A. Ordinal networks from time series}

An ordinal network ${ }^{8}$ is built from a univariate time series by mapping the data to a set of ordinal symbols as per the method of Bandt and Pompe, ${ }^{9}$ then treating each symbol as a node in a network, and assigning edges based on temporal succession in the symbolic dynamics. Explicitly, a time series $\mathbf{x}=\left\{x_{n}\right\}_{n=1}^{N}$ is embedded in $m$ dimensional space with lag $\tau$ to obtain the set of embedding vectors $\left\{\mathbf{z}_{n}\right\}_{n=1}^{N-(m-1) \tau+1}$ where $\mathbf{z}_{n}=\left(x_{n}, x_{n+\tau}, x_{n+2 \tau}, \ldots, x_{n+(m-1) \tau}\right)$. The ordinal symbolic dynamics $\mathbf{S}=\left\{S_{n}\right\}_{n=1}^{N-(m-1) \tau+1}$ is constructed by mapping each $\mathbf{z}_{n}$ to the $m$-tuple $S_{n}=\left(\pi_{1}, \pi_{2}, \ldots, \pi_{m}\right)$ where $\pi_{k} \in\{1,2, \ldots, m\}$ and $\pi_{k} \neq \pi_{l} \Longleftrightarrow k \neq l$ such that $\pi_{k}<\pi_{l}$ $\Longleftrightarrow x_{k}>x_{l}, \forall x_{k}, x_{l} \in \mathbf{z}_{n}$. If $\mathbf{z}_{n}$ contains repeated elements $x_{k}=x_{l}$ for $k \neq l$, then $\pi_{k}<\pi_{l} \Longleftrightarrow k>l$. The ordinal network is defined by the adjacency matrix $A$. Each distinct $m$-tuple that occurs in $\mathbf{S}$ corresponds to a node in $A$ with a unique integer label $\in\{1, \ldots, V\}$ where $V$ is the total number of distinct symbols in $\mathbf{S}$. Each directed edge $a_{i, j}$ is weighted based on the number of times that the forward time transition occurs between the pair of symbols in $\mathbf{S}$ corresponding to nodes $i$ and $j$, respectively.

In this paper, we consider the cases $\tau=1$ and $\tau>1$ as distinct variants of the ordinal network mapping procedure. On the one hand, ordinal symbolic methods were first conceived without a lag variable ${ }^{9}$ and the elegance of a single parameter approach to nonlinear time series analysis is undeniable. However, using time lagged ordinal symbols, as first proposed by Cao et al. ${ }^{16}$ enables the symbolic dynamics to encode information about trajectories on longer time scales while maintaining smaller $m$. The lag parameter also appears to maximise the encoding of dynamical information for specific time scales when it is set appropriately. ${ }^{17}$

\section{B. Random walks on ordinal networks}

The information encoded in an ordinal network can be used to build a state space model of $\mathbf{S}$ where, in the simplest conception, transitions from node $i$ to node $j$ are governed by a probability mass function with

$$
\operatorname{Pr}(i, j)=\frac{a_{i, j}}{\sum_{k} a_{i, k}} .
$$

Using this model, one can select an initial node on the network at random and perform a Markovian random walk to generate a new symbolic sequence $\hat{\mathbf{S}}$ of length $L$. However, when $\tau>1$ this simple model is not strictly true to the transitional properties of ordinal symbolic dynamics. To explain this, we shall first consider the case for $\tau=1$. The temporally adjacent ordinal symbols $S_{n}$ and $S_{n+1}$ correspond to the embedding vectors $\mathbf{z}_{n}=\left(x_{n}, x_{n+1}, \ldots, x_{n+(m-1)}\right)$ and $\mathbf{z}_{n+1}=\left(x_{n+1}, x_{n+2}, \ldots, x_{n+(m)}\right)$, respectively, which have $(m-1)$ overlapping elements from $\mathbf{x}$. Therefore, it must hold that $\phi\left(S_{n}(2, \ldots, m)\right)=\phi\left(S_{n+1}(1, \ldots, m-1)\right)$ where $S_{k}(i, \ldots, j)=\left(\pi_{1}, \ldots, \pi_{m-1}\right)$ (e.g., the ordered set of elements from $S_{n}$ for indices $\left.i, \ldots, j\right)$ and the operator $\phi$ gives the amplitude rank of the elements in a set (or equivalently the rank ordering). For example, the symbol $\{3,1,2\}$ can only be followed by $\{1,2,3\},\{1,3,2\}$, or $\{2,3,1\}$. We call these the allowable ordinal transitions. The procedure by which edges are allocated in an ordinal network guarantees that a Markovian random walk can only ever produce allowable ordinal transitions when $\tau=1$.

Now consider the case for $\tau>1$. Temporally adjacent ordinal symbols $S_{n}$ and $S_{n+1}$ correspond to the embedding vectors $\mathbf{z}_{n}=\left(x_{n}, x_{n+\tau}, x_{n+2 \tau}, \ldots, x_{n+(m-1) \tau}\right)$ and $\mathbf{z}_{n+1}=\left(x_{n+1}\right.$, $\left.x_{n+1+\tau}, x_{n+1+2 \tau}, \ldots, x_{n+1+(m-1) \tau}\right)$ which contain no overlapping elements from $\mathbf{x}$. In fact, the ordinal symbol $S_{n}$ places constraint not on its temporal neighbour $S_{n+1}$ but rather on the symbol $S_{n+\tau}$. To ensure that this constraint holds for a random walk on the network, the walk algorithm must "look back" $(\tau-1)$ steps from the current node to determine which of the connected nodes, if any, constitute an allowable ordinal transition. For simplicity, assume that the time index of a random walk $\hat{\mathbf{S}}$ at the current node $i$ is $(n-1)$. The transition from node $i$ to an arbitrary node $j$ is an allowable ordinal transition with respect to an arbitrary ordinal symbolic dynamics of embedding dimension $m$ if and only if $\phi\left(\hat{S}_{n-\tau}(2, \ldots, m)\right)=\phi\left(\hat{S}_{n}(1, \ldots, m-1)\right)$. It should be apparent that an initial trajectory $\hat{\mathbf{S}}_{\text {initial }} \in \mathbf{S}$ of length $L_{\text {init }}=\tau$ must be specified for a random walk which incorporates a memory of the past $\tau$ states.

We now define our algorithm which generates a random walk $\hat{\mathbf{S}}$ of length $L$ steps on the network $A$ such that allowable ordinal transitions are enforced. For the remainder of the paper, we shall refer to this algorithm as the constrained random walk. The algorithm starts by selecting an initial trajectory $\hat{\mathbf{S}}(1, \ldots, \tau)$ randomly from $\mathbf{S}$. Note that here and in the pseudo-code to follow, parenthesis immediately following an ordered set denotes an index or indices to an element or an ordered set of elements in that set, respectively, and 
hence, for example, $\hat{\mathbf{S}}(b)=\hat{S}_{b}$ and $\mathbf{S}(b, \ldots, c)=\left\{S_{b}, \ldots, S_{c}\right\}$. The step index of the walk is stored in the pointer variable $P T R$ which is initialised to $\tau$. The algorithm then walks stepwise on the network from node $i$ at $P T R$ to node $j$ at $P T R+1$ based on the transitional probabilities as given by the edges $a_{i, j}$ and conditional on the set of allowable transitions from $\hat{\mathbf{S}}(P T R-\tau+1)$. If there are no allowable edges $a_{i, j}$, then the algorithm steps backwards. A simple blocking procedure is implemented using the array variable $B L K_{n}$ such that the walk will trace backwards from a proverbial dead-end state to a node from which an allowable path can be found and then step forward again without the possibility of walking back into the same dead end until it reach $L$ steps and returns $\hat{\mathbf{S}}$. If a path cannot be found, then the algorithm will break and exit. ${ }^{18}$ The pseudo-code for our algorithm is as follows:

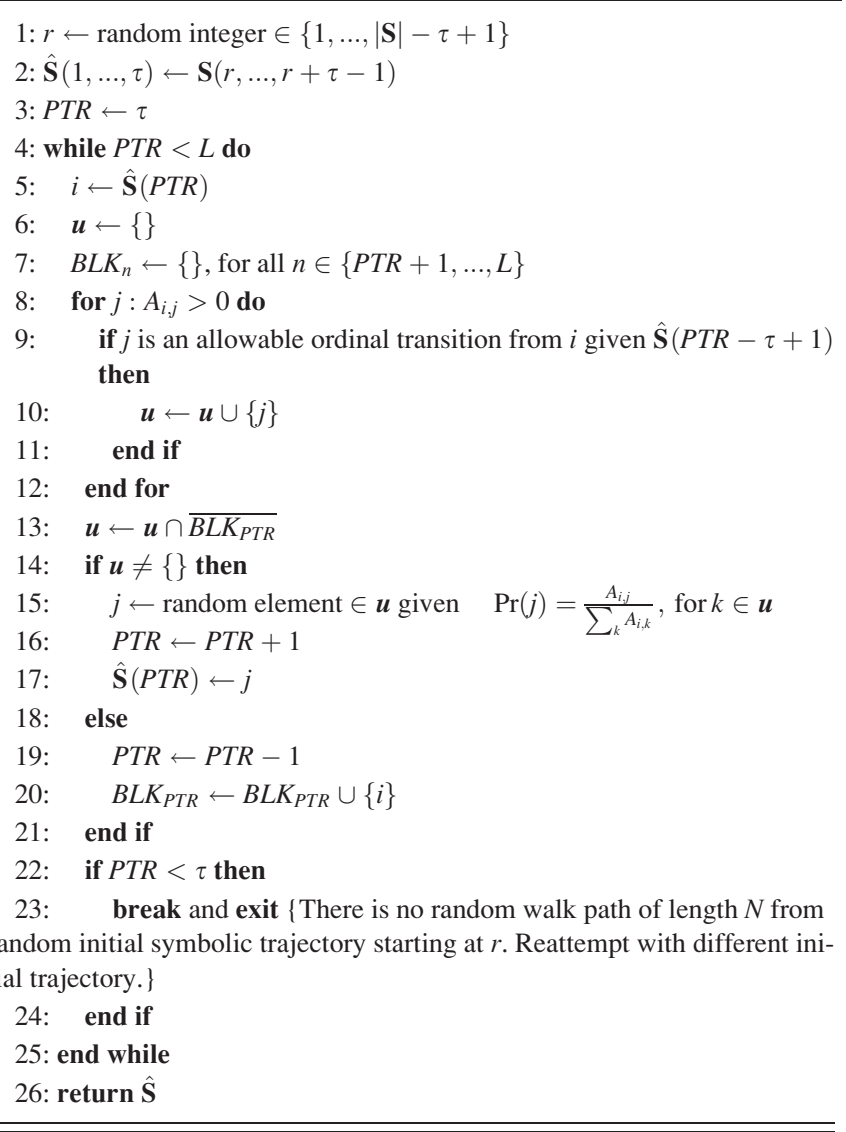

\section{Regenerating time series from random walks}

To regenerate a time series from the random walk symbolic dynamics $\hat{\mathbf{S}}$, we randomly reassign data from the original time series based on the ordinal symbolic map as follows: For all $n \in 1, \ldots L$, we find all embedding vectors $\mathbf{z}_{k}$ from the original time series $\mathbf{x}$ which map to symbol $\hat{S}_{n}$. We then select one of these $\mathbf{z}_{k}$ at random and assign its first element to time index $n$ such that $\hat{x}_{n}=x_{k}$, where $\hat{\mathbf{x}}=\left\{\hat{x}_{n}\right\}_{n=1}^{L}$ is the regenerated time series. Assignment of $x_{k}$ from $\mathbf{z}_{k}$ is performed with replacement, which is to say, that $x_{k}$ can be assigned multiple times in $\hat{\mathbf{x}}$ when it may only appear once in $\mathbf{x}$. The regenerated data $\hat{\mathbf{x}}$ can be considered as a type of surrogate time series ${ }^{6,19}$ for $\mathbf{x}$ based on the ordinal network model.

\section{QUALITATIVE RESULTS}

Figure 1 shows the complete process of taking time series data, shown as time delay reconstruction of the attractor in this instance, then mapping that data to an ordinal network and, finally, regenerating a surrogate time series using a constrained random walk and data reassignment. The data used are a discrete sampled time series from a continuous chaotic Lorenz system. Note that all the time series used in this study have been generated by numerically solving their respective systems using a fourth-fifth order Runge-Kutta algorithm. It can be observed in Figure 1(b) that the ordinal network encodes topological features of the attractor including the two wings and the separatrix. Visual inspection of the reconstructed attractor for the surrogate time series, as shown in Figure 1(c), suggests that the ordinal network mapping and the random walk constitute a good stochastic approximation of the temporal progression of states which manifest in the original dynamics, with the exception of the slightly jagged and noisy appearance of the trajectories. The topology of the surrogate attractor is enforced by the data reassignment process because all points in $\hat{\mathbf{x}}$ occur somewhere in $\mathbf{x}$. However, the random walk and the randomness in the data reassignment procedure do not guarantee that the density of an attractor reconstructed from the surrogate time series will be consistent with the original attractor. In the case of Figure 1(c), to the extent that the density of the surrogate attractor appears similar to the original, we can infer that the constrained random walk has sampled the attractor in a manner that is relatively consistent with the original dynamics, or alternatively that the random walk symbolic dynamics $\hat{\mathbf{S}}$ has a stationary distribution similar to that of the original symbolic dynamics $\mathbf{S}$. This can be quantified by the correlation integral which we compute to estimate the correlation dimension in Section V B.

Figure 2 shows surrogate Lorenz time series for various $m$ using constrained random walks on ordinal networks with $\tau=7$, and Markovian random walks on ordinal networks with $\tau=1$, respectively. For the case of $\tau=7$ with constrained random walks (Figure 2(a)), the surrogate time series for $m=7$ and $m=10$ are arguably pseudo-periodic but they do not appear to be useful stochastic approximations of the original system. This is the result of degeneracies which arise when the partition defined by the ordinal map is not sufficiently fine to capture the complexity of the dynamics. For example, if the total time span of a symbol given $m$ and $\tau$ is shorter in duration than a segment of the Lorenz time series within which the trajectory is cycling around only one of the wings on the attractor, then the ordinal symbolic dynamics $\mathbf{S}$ will not distinguish between the two wings. Evidently, this will not only affect both the map from $\mathbf{x}$ to $\mathbf{S}$ and the reassignment from $\hat{\mathbf{S}}$ to $\hat{\mathbf{x}}$ but also diminish the quality of the temporal structure encoded in the network and, hence, affect the random walk. Information is lost at each of these steps and the resulting model is less accurate (e.g., both the random walk and the data reassignment procedure can generate trajectories that spuriously jump between the wings of the attractor). By increasing $m$, the ordinal partition becomes finer, and for $m=13,16$ and 20 in Figure 2(a), the 


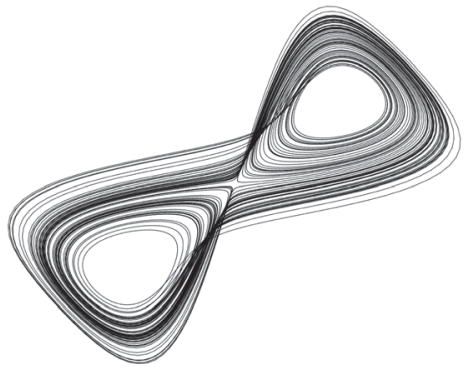

(a)

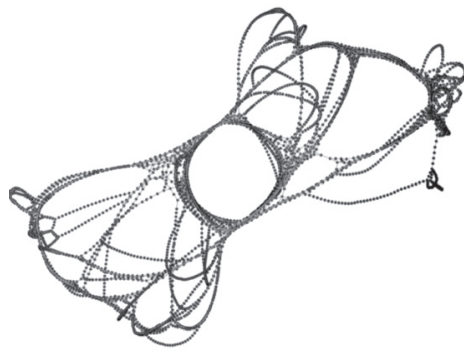

(b)

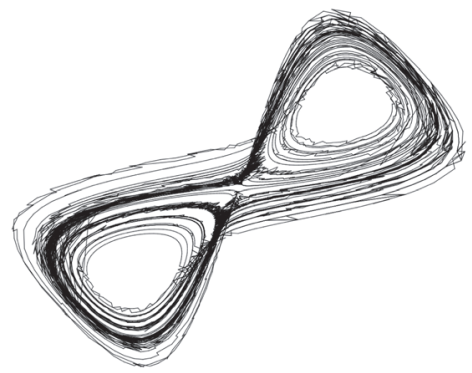

(c)
FIG. 1. (a) A time delay reconstruction of the Lorenz attractor using the $x$ component time series with embedding dimension $m=3$ with embedding lag $\tau=7$. The time series comprises 10000 equi-spaced observations with sampling time-step $d t=0.01$ (approximately 100 points per mean cycle). (b) The ordinal network constructed from the same Lorenz time series using embedding dimension $m=16$ and embedding lag $\tau=7$. This visualisation was produced using a springelectrical embedding algorithm in Wolfram Mathematica. (c) The time delay reconstruction of a stochastic approximation of Lorenz system generated by taking a constrained random walk on the ordinal network and assigning corresponding time series points from the original data. surrogate time series appear to be good stochastic approximations of the deterministic chaotic flow data.

The case for Markovian walks with $\tau=1$ is similar, as shown in Figure 2(b). For $m=7$ and $m=10$, the surrogate time series are characterised by what appears to be pseudoperiodic oscillations with little or no distinction between the two wings of the Lorenz attractor. The time series appear closer to the original data as $m$ increases and the model provides a good stochastic approximation of the deterministic dynamics when $m=20$.

\section{RESULTS FOR ORDINAL NETWORKS WITH $\tau>1$}

In this section, we investigate surrogate time series that are regenerated from ordinal networks with a time lag parameter $\tau>1$ using the constrained random walk algorithm. This investigation is executed in two subsections, each with a distinct purpose. First, in Section IV A, we compare the original ordinal symbolic dynamics $\mathbf{S}$ with random walk symbolic dynamics $\hat{\mathbf{S}}$ using order recurrence plots and order recurrence quantification analysis. We do this to
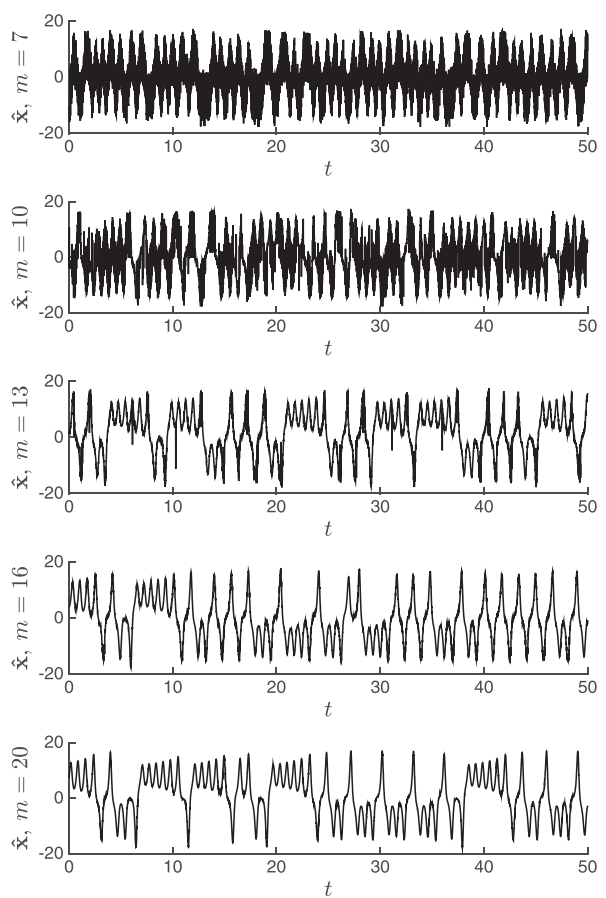

(a)
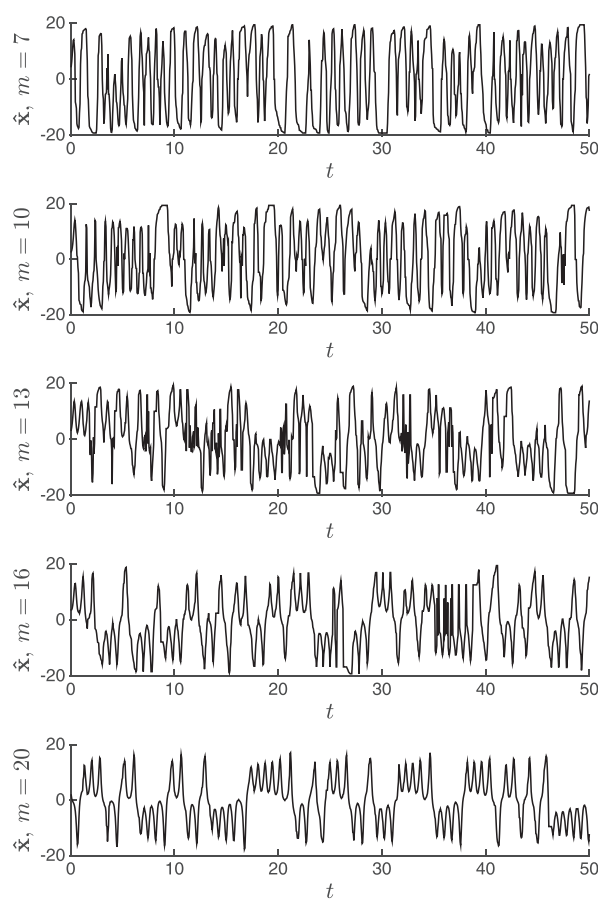

(b)
FIG. 2. (a) Surrogate time series $\hat{\mathbf{x}}$ for the $x$-component of the Lorenz system regenerated using constrained random walks on ordinal networks with embedding lag $\tau=7$ and various embedding dimensions $m$. The time series used to construct the ordinal network comprises 10000 equi-spaced observations with sampling time-step $d t=0.01$ (approximately 100 points per mean cycle). (b) Surrogate time series $\hat{\mathbf{x}}$ for the $x$-component of the Lorenz system regenerated using Markovian random walks on ordinal networks with embedding lag $\tau=1$ and various embedding dimensions $m$. The time series used to construct the ordinal network comprises approximately 400000 equi-spaced observations with sampling time-step $d t=0.05$ (approximately 20 points per mean cycle). 
determine how well ordinal networks and constrained random walks can preserve the temporal structure of the symbolic dynamics. Second, in Section IV A, we investigate how effectively invariant properties are preserved through the complete process of mapping a time series to an ordinal network and then regenerating a surrogate. Specifically, we estimate the largest Lyapunov exponent for the original time series $\mathbf{X}$ and a set of surrogate time series $\hat{\mathbf{x}}$.

Both analyses in this section pertain to a single $x$-component time series from the Lorenz system in the archetypal chaotic regime with parameters $\rho=28, \sigma=10$, and $\beta=8 / 3$. The time series comprises 10000 equi-spaced observations with sampling time-step $d t=0.01$ which results in approximately 100 points per mean cycle. Transients have been removed.

\section{A. Order recurrence plots and recurrence quantification analysis}

An order recurrence $\operatorname{plot}^{13,14}$ (ORP) is defined by the matrix:

$$
\mathbf{R}_{n, k}=\left\{\begin{array}{ll}
1 & \text { if } S_{n}=S_{k} \\
0 & \text { if } S_{n} \neq S_{k}
\end{array}, \text { for } n, k=1, \ldots, L,\right.
$$

where $S_{n}$ and $S_{k}$ are symbols in the ordinal symbolic dynamics $\mathbf{S}$ of length $L$. This is to say, that ORPs are equivalent to traditional recurrence plots ${ }^{20}$ except that rather than applying a threshold to an appropriate distance metric to define a recurrence between states, the state of the system at time index $n$ is considered to have a recurrence in an ORP at time index $k$ if the states at $n$ and $k$ map to the same ordinal symbol. The primary reason we employ ORPs in this study is not to attempt analysis or classification of time series. Instead, we use them as a visual and quantitative tool for comparing the recurrence properties of the symbolic dynamics $\mathbf{S}$ from the original Lorenz time series $\mathbf{x}$ and symbolic dynamics $\hat{\mathbf{S}}$ that are generated by the constrained random walk algorithm.

Figure 3 shows the ORPs for $\mathbf{S}$ and $\hat{\mathbf{S}}$, respectively, each with length $L=9896$. We elected to use embedding dimension $m=16$ based on our qualitative analysis of constrained random walk time series in Section III. Both ORPs are characterised by complex structures of short diagonal lines of various lengths which are parallel to the line of identity indicating the possibility of deterministic quasiperiodic dynamics. ${ }^{14}$ These structures tend to fall into epochs that are disrupted where the time series spirals outward around one of the unstable foci for at least several cycles. For example, see the white bands at $n \approx 4000$ and 5000 in the ORP for the original time series and $n \approx 1800$ and 6000 in the ORP from the constrained random walk. In the ORP for the original time series, the epochs appear to be larger and comprise more complex structures than those which occur in the ORP for the random walk. However, there are some small scale structures which are seemingly identical in both ORPs. For example, see the small structure at $(n, k) \approx(5000,5000)$ in the ORP for the original time series and $(n, k) \approx(6000,6000)$ in the ORP from the constrained random walk.

Recurrence quantification analysis (RQA) measures have been computed on the ORPs for $\mathbf{x}$ and 10 realisations of random walks $\hat{\mathbf{S}}$ for $m=3, \ldots, 20$. The results were validated using the Cross Recurrence Plot Toolbox Version $5.21(\mathrm{R} 31 \mathrm{c})^{14}$ and are shown in Figure 4. The recurrence rate of $\hat{\mathbf{S}}$ is very close to that of $\mathbf{S}$ for all $m$ with negligible variance between the realisations of the random walk. The two measures of vertical line structures-the laminarity and trapping time-are also in close agreement between the original time series and the constrained random walks, albeit with more variance in these measures for $\hat{\mathbf{S}}$. This is also the case for the entropy of the distribution of diagonal line lengths. Furthermore, determinism and average diagonal line length indicate that diagonal line structures are relatively consistent between $\mathbf{S}$ and $\hat{\mathbf{S}}$ for smaller $m$. However, these measures suggest significant differences in the dynamics for $m \gtrsim 15$. We postulate that the divergence of $D E T$ and $L$ is likely a result of the ordinal network model breaking down due to the finiteness of data with respect to $m$. As $m$ increases, the number of distinct ordinal symbols encoded from the time series begins to approach the total length of the time series. Therefore, the amount of available data no longer guarantees good sampling of the transitional dependencies between symbolic states.

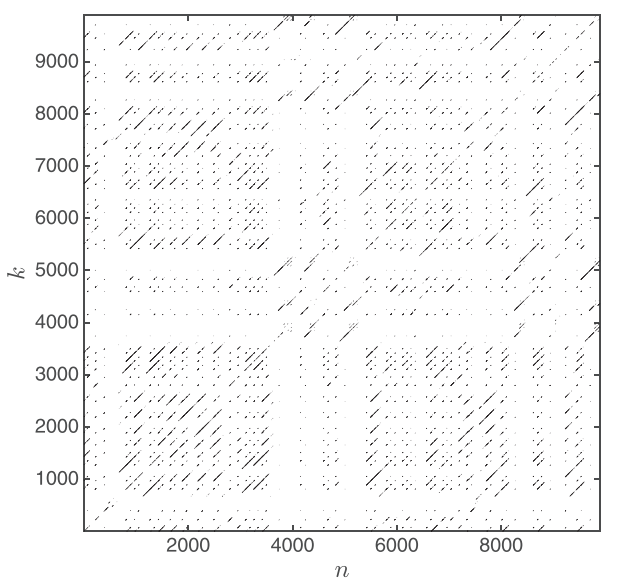

(a)

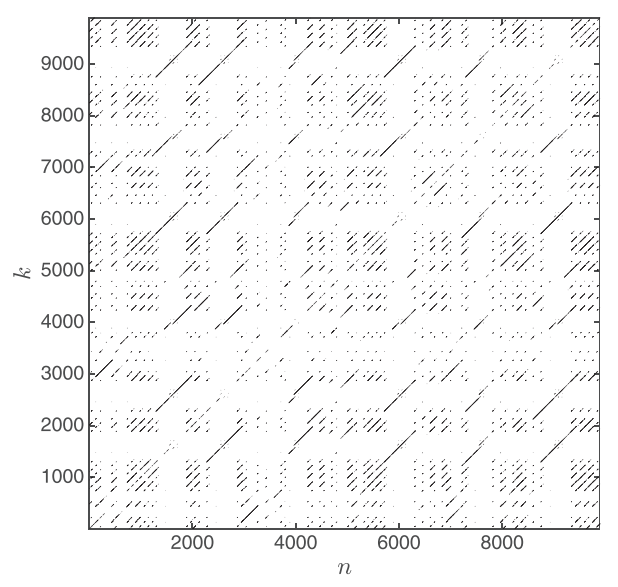

(b)
FIG. 3. Order recurrence plots for (a) ordinal symbolic dynamics $\mathbf{S}$ for the chaotic Lorenz time series as computed for $m=16$ and $\tau=7$ and (b) a single realisation of a constrained random walk symbolic dynamics $\hat{\mathbf{S}}$ from the corresponding ordinal network model. A recurrence (black) is marked at coordinate $(n, k)$ if the ordinal symbol at time index $n$ recurs at time index $k$ (see Equation (2)). 

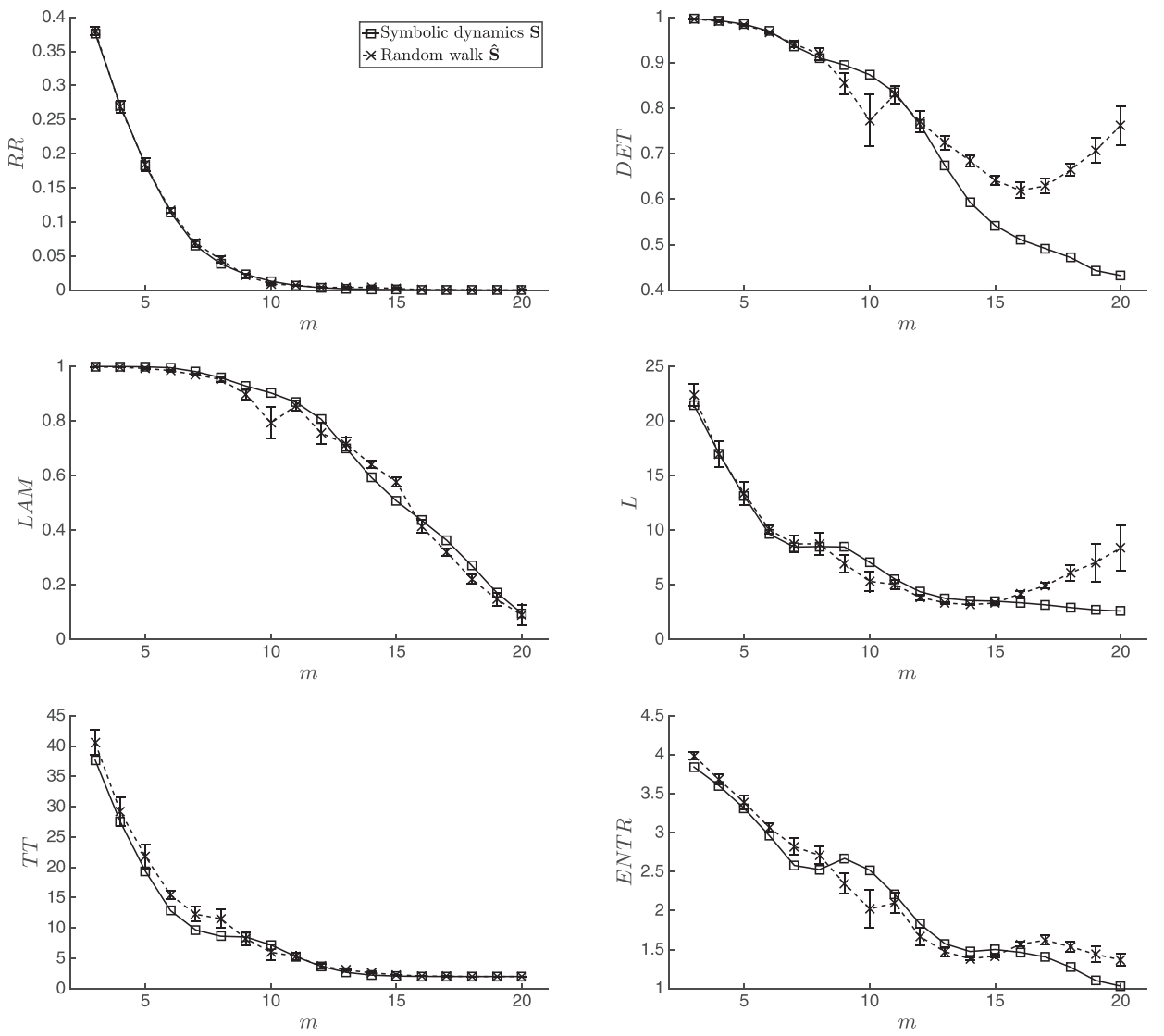

FIG. 4. Recurrence quantification analysis measures plotted against embedding dimension $m$ with $\tau=7$ for ordinal symbolic dynamics $\mathbf{S}$ from the original chaotic Lorenz time series (solid lines) and constrained random walk symbolic dynamics $\hat{\mathbf{S}}$ from the corresponding ordinal network model (dashed lines). Each data point for the $\hat{\mathbf{S}}$ curves is the sample mean of the measure for 10 realisations of the random walk. Error bars give the standard deviation. The measures computed are the recurrence rate $(R R)$, determinism (DET), laminarity $(L A M)$, average diagonal line length $(L)$, trapping time (TT), and the Shannon entropy of the distribution of diagonal line lengths $(E N T R)$.
Our results have shown that the RQA measures for recurrence rate and vertical line structures are generally consistent between the original symbolic dynamics and the constrained random walks. Measures of diagonal line structures and the qualitative dissimilarities evident in the ORPs reveal differences in the dynamics between the deterministic chaotic flow generated by the Lorenz system and the stochastic approximation encoded in the ordinal network. However, the ORP for the constrained random walk is not vastly different to the original and still exhibits structures that would usually indicate strong quasi-periodic determinism.

\section{B. Estimation of Lyapunov exponents (I)}

Figure 5 shows the logarithm of the average separation of nearby trajectories in the reconstructed phase space for the original time series $\mathbf{x}$ and a single surrogate realisation $\hat{\mathbf{x}}$ of length $L=10000$. Note that we have used the TISEAN software package ${ }^{21}$ for this computation and those which follow in Sections VA and VB. Under the assumptions of determinism and ergodicity, the existence of a positive linear scaling region that is invariant to the embedding dimension of the reconstructed phase space is evidence of the exponential divergence of trajectories, where the gradient of this region corresponds to the largest Lyapunov exponent. ${ }^{22}$ As expected, a scaling region is clearly observable for $\mathbf{x}$ and spans approximately $1 \leq \Delta t \leq 4$. A scaling region of about half this time span can also be observed for $\overline{\mathbf{x}}$. The surrogate time series is not deterministic; therefore, it would not be correct to infer the identification of chaotic exponential separation of trajectories. However, the presence of a well defined scaling region in $\hat{\mathbf{x}}$ with a gradient very similar to that which was computed for $\mathbf{x}$ demonstrates that the ordinal network and constrained random walk are partially preserving dynamical properties which relate to invariant measures on the original system.

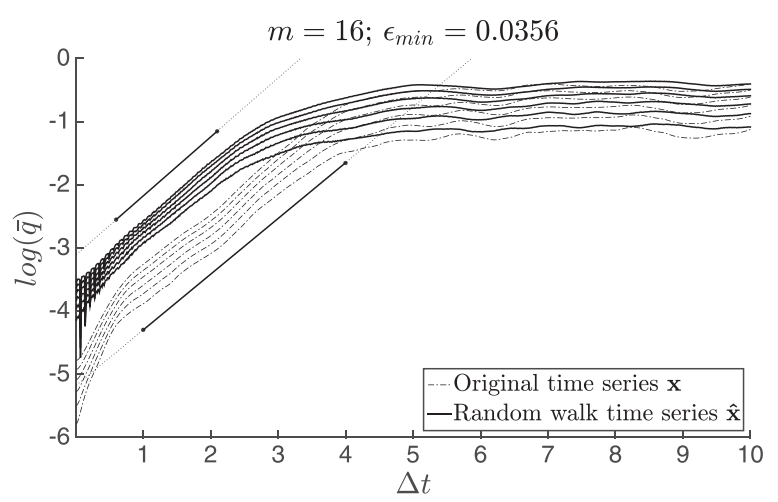

FIG. 5. The average separation of nearby trajectories $\bar{q}$ against $\Delta t$ in the reconstructed attractors of the original chaotic Lorenz time series $\mathbf{x}$ and a single realisation of a constrained random walk surrogate time series $\hat{\mathbf{x}}$ for the estimation of the largest Lyapunov exponent $\lambda_{1}$ by the Rosenstein algorithm. ${ }^{22}$ The random walk time series $\hat{\mathbf{x}}$ was generated by an ordinal network model of the Lorenz time series using $m=16$ and $\tau=7$. For both time series, $\bar{q}$ is plotted for $\tau=7$ and $d=3,4,5,6,7,8$ (from lowest to highest curve in each set respectively) where $d$ is the dimension in which $\mathbf{x}$ and $\hat{\mathbf{x}}$ are embedded for the computation of the separation of trajectories. The minimum spatial scale on which the algorithm searches for neighbours is given by $\epsilon_{\min }$. The estimates for $\lambda_{1}$ are given by the gradients of the dotted lines where the lower line corresponds to $\mathbf{x}$ and the upper line to $\hat{\mathbf{x}}$. These lines are computed using linear regression based on the points in the linear scaling regions of $\bar{q}$ which are demarcated by the solid potions of each respective line of best fit. 
Apart from the length of the scaling region, the only other difference of note between the curves in Figure 5 for $\mathbf{x}$ and $\hat{\mathbf{x}}$ is the average initial separation distance. This is likely due to an increased degree of heterogeneity in the reconstructed attractor for $\hat{\mathbf{x}}$ than for $\mathbf{x}$ which results from the random walk. The random walk will be inherently more likely to follow the symbolic trajectories that occur more frequently in the original time series. Therefore, the reconstructed attractor for the surrogate time series will trace the denser areas of the original attractor but visit the less dense areas with lower probability than would occur in the true dynamics. This is can be observed in Figure 1 under close inspection. The topology of the attractor may be such that the Rosenstein algorithm ${ }^{22}$ needs to search within a larger distance on average to find a sufficient number of neighbouring states in phase space for the robust estimation of the separation of trajectories and, therefore, the average initial separation of points in the neighbourhood may be larger.

We repeated the estimation of $\lambda_{1}$ for 10 realisations of $\hat{\mathbf{x}}$ of length $L=10000$ from constrained random walks on ordinal networks that were constructed from the Lorenz time series using parameters $m=8,10,12,14,16$ and $\tau=7$. To reiterate, $\lambda_{1}$ can only be interpreted as an estimate of the largest Lyapunov exponent for the original time series $\mathbf{x}$. For $\hat{\mathbf{x}}$ it is simply an estimate of the average rate of divergence nearby trajectories in a given realisation of the time series. We report that no clear linear scaling region can be identified for $m<12$. Furthermore, we report that the length of the scaling region for $\hat{\mathbf{x}}$ is consistently smaller than for $\mathbf{x}$ but tends to increase with $m$. Table I shows that where a linear scaling region could be identified $(m=12,14,16)$ the mean estimate of $\lambda_{1}$ is close to the value estimated for the original time series and variance between the 10 realisations is relatively small. The length of scaling region for these estimates was relatively consistent and generally spanned 1 to 1.5 units. In summary, we find that the ordinal network model produces stochastic time series which can approximate the exponential separation of trajectories when $m$ is sufficiently large.

\section{RESULTS FOR ORDINAL NETWORKS WITH $\tau=1$}

In this section, we explore the properties of the networkbased surrogate time series generated by means of the Markovian random walk $(\tau=1)$. Characteristic features of 10 surrogate time series $\hat{\mathbf{x}}^{(\mathbf{m})}$ for each $m=7,10,13,16,20$

TABLE I. Estimates of the largest Lyapunov exponent $\lambda_{1}$ as per Figure 5 for the original chaotic Lorenz time series $\mathbf{x}$ and 10 realisations of a constrained random walk surrogate time series $\hat{\mathbf{x}}$. The random walk time series $\hat{\mathbf{x}}$ were generated by an ordinal network model of the Lorenz time series using $m=12,14,16$ and $\tau=7$. All time series were embedded with $d=8$ and $\tau=7$ for the computation of $\lambda_{1}$.

\begin{tabular}{llll}
\hline \hline Original time series $\mathbf{x}$ & & \multicolumn{2}{c}{$\lambda_{1}=0.8826$} \\
\cline { 1 - 1 } Random walk time series (10 realisations) & & $\operatorname{Mean}\left(\lambda_{1}\right)$ & $\operatorname{Var}\left(\lambda_{1}\right)$ \\
\hline$\hat{\mathbf{x}}$ for $m=12$ & & 0.8298 & 0.0009 \\
$\hat{\mathbf{x}}$ for $m=14$ & & 0.7250 & 0.0069 \\
$\hat{\mathbf{x}}$ for $m=16$ & 0.9784 & 0.0024 \\
\hline \hline
\end{tabular}

from an original time series $\mathbf{x}$ are examined using three different tools, each with a distinct aim in mind. First, in Section VA, estimates of the largest Lyapunov exponent $\left(\lambda_{1}\right.$; algorithm by $\left.\mathrm{Kantz}^{23}\right)$ are calculated in order to assess the local divergence of trajectories in the surrogate state space. Second, in Section V B, fractal dimension is estimated based on the implementation of the correlation $\operatorname{sum}^{24}$ to evaluate the degree of consistency of the reconstructed attractor's density with the original. Finally, in Section V C, recurrence quantification analysis is applied to the associated recurrence plots. ${ }^{14}$

To demonstrate the properties of surrogate realisations originating from transition rules on this type of networks, we examine the scalar $x$-component time series generated by the Lorenz equations in the chaotic regime as per Section IV. An original time series $\mathbf{x}$ of length $N=400000(\sim 20000$ cycles) is generated. The continuous dynamics are sampled at $d t=0.05$. The mean cycle time is $\mathcal{T}_{M}=0.95$ hence the sampling rate corresponds to approximately 20 points per cycle.

As a first comparison between the random surrogate time series and the original deterministic trajectories, we estimated the time-delayed mutual information (MI) for each of the 10 different $\hat{\mathbf{x}}^{(\mathbf{m})}$ and the original $\mathbf{x}$. Table II presents the average value (over all 10 realisations) of its first minimum, $\mathrm{MI}_{\min }$. Nonzero standard deviations are shown wherever appropriate. We observe a relative consistency among the 10 different realisations-as indicated by the low standard deviations. The mean $\mathrm{MI}_{\min }$ is close to the value computed from $\mathbf{x}$ when $m \geq 16$ which is approximately three quarters of a quarter period.

\section{A. Estimation of Lyapunov exponents (II)}

Local exponential divergence between trajectories was calculated for ten different spatial scales (with the minimum corresponding to $\frac{1}{1000}$ and the maximum to $\frac{1}{50}$ of the range of the data) and seven different values for the embedding dimension $d=4,5, \ldots, 10$. Figure 6 depicts plots of the logarithm of the divergence in state space for $\hat{\mathbf{x}}^{(13)}, \hat{\mathbf{x}}^{(16)}$, and $\hat{\mathbf{x}}^{(20)}$. Estimates shown correspond to a single spatial scale and realisation, for separation times $\Delta t \in[0,10]$ which is approximately equal to 10 cycles in the original system. Note that results are consistent among all realisations and all scales larger than a certain required minimum (which is slightly greater than the corresponding threshold in the original data).

TABLE II. Mean and standard deviation (wherever nonzero) of the first minimum of the time-delayed mutual information for the original and network-based surrogate time series.

\begin{tabular}{lc}
\hline \hline & MI $_{\min }$ \\
\hline $\mathbf{x}$ & 4 \\
$\hat{\mathbf{x}}^{(7)}$ & $15 \pm 2.83$ \\
$\hat{\mathbf{x}}^{(10)}$ & 13 \\
$\hat{\mathbf{x}}^{(13)}$ & 11 \\
$\hat{\mathbf{x}}^{(16)}$ & 5 \\
$\hat{\mathbf{x}}^{(20)}$ & 5 \\
\hline \hline
\end{tabular}



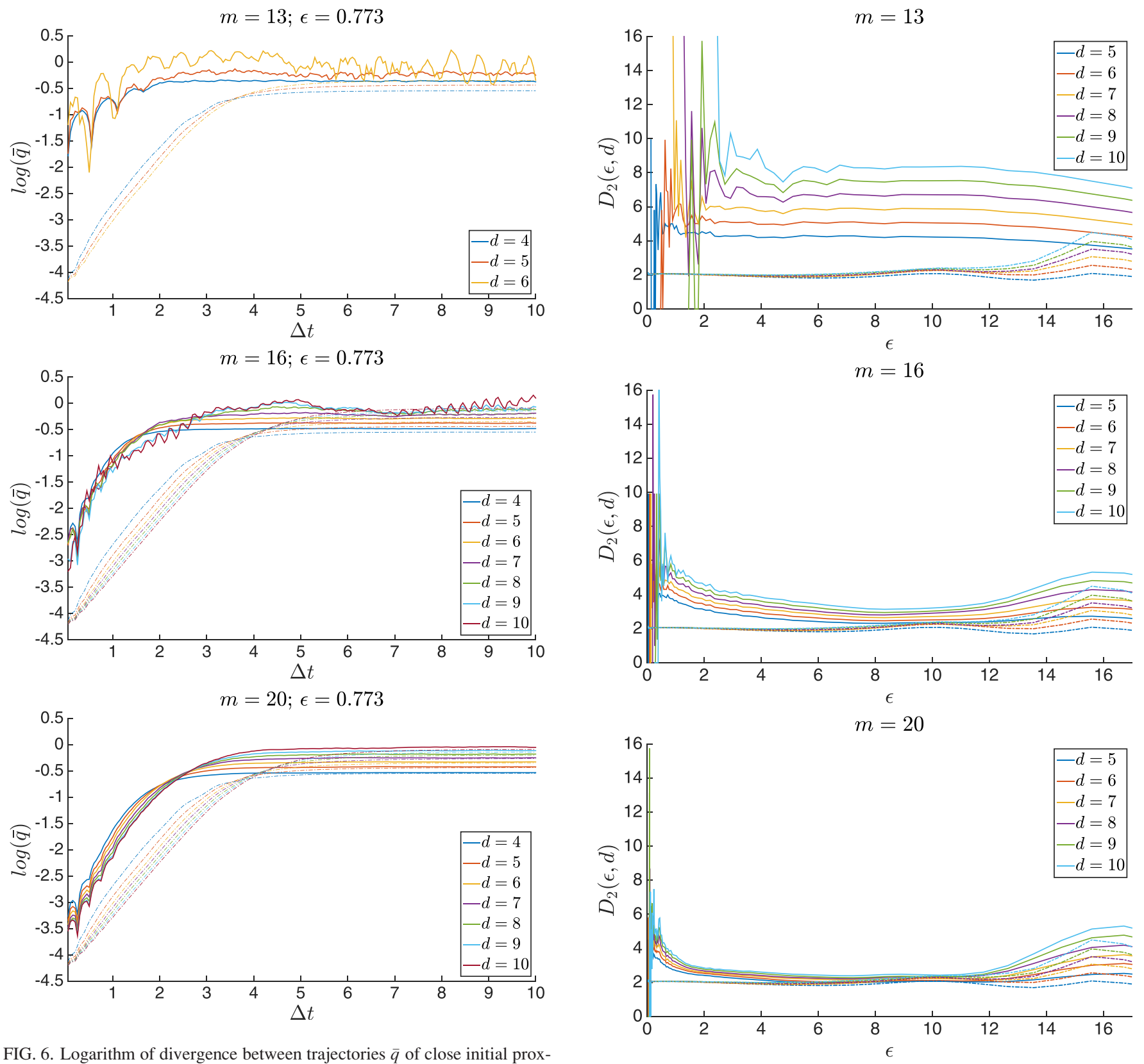

FIG. 6. Logarithm of divergence between trajectories $\bar{q}$ of close initial proximity for the original time series $\mathbf{x}$ (dashed lines) and the Lorenz networkbased surrogates $\hat{\mathbf{x}}^{(m)}$ (solid lines) with $m=13,16$, and 20 for various embedding dimensions $d$ and a selected representative spatial scale $\epsilon$. Results were computed using the Kantz algorithm. ${ }^{23}$

First of all, observe the saturation of the divergence to a certain average value at larger spatial scales $\epsilon$. This value reflects the size of the associated state-space attractors since average divergence over all trajectories should saturate to a limiting value related to the maximum possible distance between any two state-space points. This is because all points in $\hat{\mathbf{x}}$ are drawn from $\mathbf{x}$ in the data reassignment procedure for the regeneration of $\hat{\mathbf{x}}$ from $\hat{\mathbf{S}}$, as explained in Section III. Larger $m$ values lead to a saturation which approximates the corresponding value for the reconstructions of the original data (see lower panel of Figure 6).

A scaling region is observed only for $m \geq 16$ and is shorter (about half) in comparison to the one present in the estimates computed from the original time series. The pattern length parameter $m$ clearly plays a crucial role, with

FIG. 7. Correlation dimension $D_{2}$ estimates for the original time series $\mathbf{x}$ (dashed lines) and the Lorenz network-based surrogates $\hat{\mathbf{x}}^{(m)}$ (solid lines) with $m=13,16$, and 20 for various embedding dimensions.

surrogate time series producing a good stochastic approximation to the original data only if $m$ is sufficiently large. The largest Lyapunov exponent, computed as the slope in these plots, is consistently higher for the surrogates. Specifically, the approximate mean slope is estimated to be $1.497 \pm 0.021$ when $m=20$-approximately 1.6 times in comparison to the value computed from the original data $(\sim 0.906)$. This implies a faster divergence of state-space trajectories, a fact in agreement with the shorter scaling region. Note also the more pronounced and distinctive effects of the random nature of the surrogates, evident in the smaller spatial scales as expected.

As discussed in Section IV B, one should not infer the existence of a Lyapunov exponent and local exponential divergence for time series which have not been generated by 
means of strict deterministic rules. Furthermore, the average separation of trajectories is faster in the random walk surrogates than for the original deterministic time series. The presence of a scaling region, however, is indicative of partial preservation of dynamical properties by the network-based surrogates. With increasing $m$ one also observes an (albeit slow) elongation of the scaling region, fewer fluctuations and saturation to a value closer to the reconstructed attractor's size for the original data. This demonstrates that for increasing $m$ the ordinal network surrogates gradually attain similar dynamical features with respect to separation of trajectories (a key characteristic of chaotic behaviour) as data generated by the original system.

\section{B. Estimation of fractal dimension}

Correlation dimension estimates of the Lorenz surrogates are depicted in Figure 7, with dotted lines representing the estimates from $\mathbf{x}$. Values $d<5$ are insufficient for accurate estimation, even from the original data, and are thus omitted. Surrogates obtained from networks with $m \leq 16$ constitute a typical case of data where no estimate should be quoted since curves behave differently. However, as $m$ increases, we observe the following interesting effect: the curves start approaching each other, especially within the scaling region. Furthermore, this region is being elongated. The result is an overestimate compared to the actual $D_{2}$ value of the system, irrespective of $m$ used. In a strict sense, the estimate is still inadequate even in the $m=20$ case, since the specific value is approximately $\simeq 2.1-2.5$ (dependent on $d$ used). Therefore, there are still significant fluctuations between the quoted values to classify this object as fractal and this holds for all $d$ values. In addition, the actual estimate is 2.05. Consequently, the density of the surrogate statespace attractor differs from the original but significant resemblance appears with increasing $m$. To explain this observation, recall the reconstructed attractors of Figure 7 . The degeneracies present due to the noisy nature of the surrogate realisations lead to an attracting object of higher fractal dimension than the original-it occupies a larger portion of the three-dimensional state-space reconstruction. Furthermore, self-similarity properties have been partially lost, especially in smaller scales. While these effects are minimised as $m$ increases, network-based surrogates produce state-space points which in collection approach a fractal set but do not necessarily form one. The actual $D_{2}$ of the system acts as a lower bound to these estimates.

\section{Recurrence quantification analysis}

RQA can reveal the capacity of ordinal network representations for efficiently encoding information about recurrence of trajectories in the underlying state space. Here, we explore the properties of recurrence plots (RPs) associated with the Markovian random walk surrogate time series computed from the Lorenz system for $m=10$ and $m=20$. In this section, we employ traditional recurrence plots where recurrence is based on a Euclidean distance metric in reconstructed phase space $^{20}$ (not order recurrence plots as were investigated in Section IV A). Six commonly employed measures for analysing the recurrence plots are shown in Figure 8. Each measure computed from an RP reflects some property of the underlying dynamical system. We examine
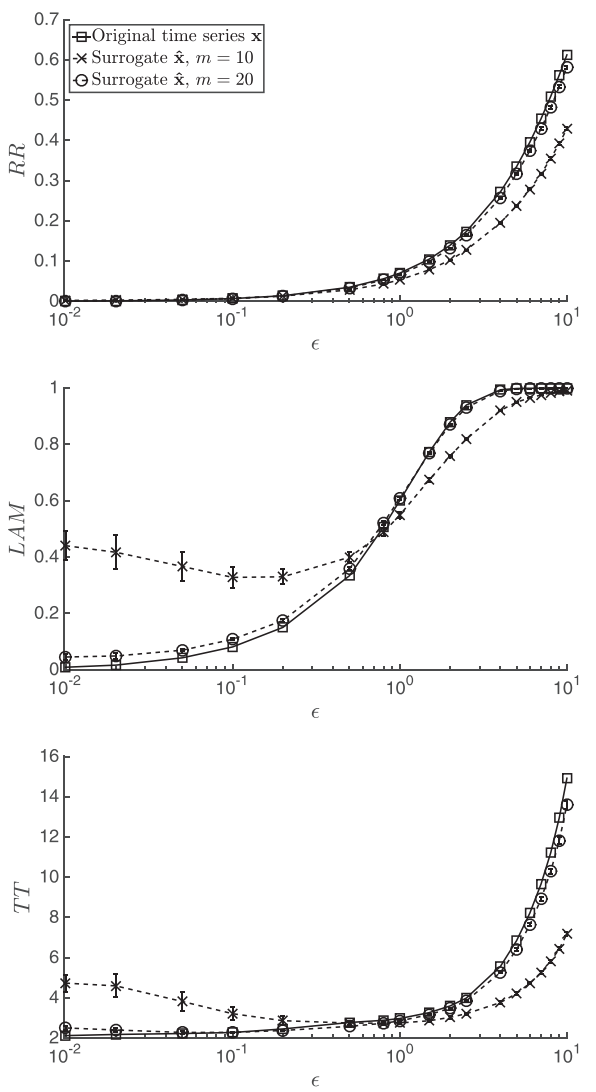
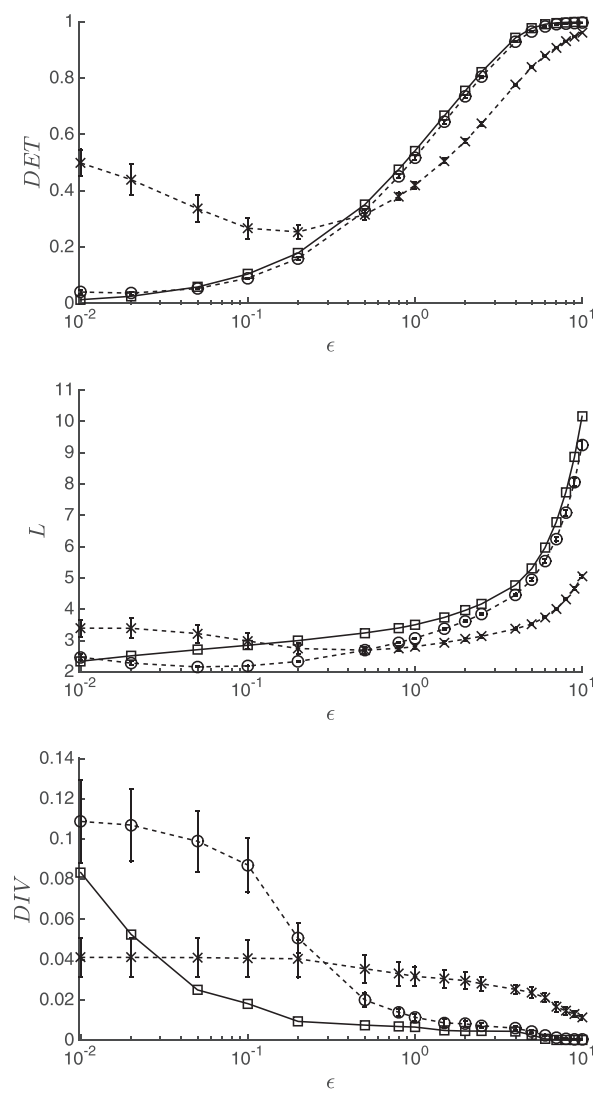

FIG. 8. Recurrence quantification analysis measures plotted against the recurrence threshold $\epsilon$ for the original time series $\mathbf{x}$ (solid lines) and 10 realisations of the Lorenz network-based surrogates $\hat{\mathbf{x}}^{(10)}$ and $\hat{\mathbf{x}}^{(20)}$ (dashed lines). Each data point for the $\hat{\mathbf{x}}$ curves is the sample mean of the measure for 10 realisations of the random walk. Error bars give the standard deviation. The measures computed are the recurrence rate $(R R)$, determinism $(D E T)$, laminarity $(L A M)$, average diagonal line length $(L)$, trapping time (TT), and divergence $(D I V)$. 
the spectrum of values for each measure across several spatial scales.

The top panels depict the recurrence rate $R R$ and determinism DET of the RP for various spatial scales. These measures correspond to the probability of recurrence of dynamical states and the predictability of the system, respectively. The $m=10$ surrogates exhibit a lower RR in comparison to $\mathbf{x}$. This indicates that surrogate time series are less likely to revisit a certain dynamical state on average in comparison to the original data. Hence, points are more sparsely dispersed in phase space. Time-delay reconstructions (see supplementary material Appendix A) confirm this hypothesis and further illustrate the lack of a compact fractal-like structure which acts as an attractor. This also holds for any $m \leq 13$ surrogates. Significantly lower values with increasing $\epsilon$ in comparison to $\mathbf{x}$ are also exhibited by the average diagonal length $L$ (implying a shorter predictability time, greater complexity, or a noisy time series) and the trapping time (average vertical length) $T T$. The latter suggests that $\hat{\mathbf{x}}^{(10)}$ are generated by dynamics whereby trajectories spend less time in a specific state, motion in state space is faster on average. This observation is further reinforced by examining the large discrepancy observed for the inverse of the maximum diagonal length, the divergence $D I V$, which is related to the largest Lyapunov exponent of the system. In particular, the consistently higher $D I V$ values computed from surrogates among all spatial scales are in full agreement with the Lyapunov exponent analysis conducted above. The plot reveals the previously shown faster local separation of proximate trajectories, especially at small scales, as well as the existence of non-negligible fluctuations among the 10 different realisations which dissipate with increasing $\epsilon$.

On the contrary, the $m=16$ and $m=20$ surrogates follow the original data more closely in terms of almost all RQA measures. As Figure 8 illustrates, the curves computed from $\mathbf{x}$ and $\hat{\mathbf{x}}^{(20)}$ coincide for all measures and, additionally, a minimal variability in these values is observed among the different realisations. A large discrepancy is found upon examination of the divergence. At all scales, divergence is greater for the surrogates, which is in accordance with the observations made by inspection of the lower panel of Figure 6. At smaller scales, the observed discrepancy is even greater. The DIV curves coincide only once $\epsilon$ is large enough (of the order of the size of the attractor) that it no longer provides a sensible parameter value for local divergence and estimates of $\lambda_{1}$. The laminarity measure $L A M$ is particularly illuminating in terms of attractor density. $L A M$ is related to the amount of laminar phases present in a dynamical flow. A high value represents a large amount of state-space trajectories whose motion organises in parallel layers without any disruptions. This notion is related to the degree of selfsimilarity that characterises a system. The original Lorenz data $\mathbf{x}$ obtain a relatively high $L A M$ even at the mesoscale. Values of $L A M$ for the surrogates $\hat{\mathbf{x}}^{(20)}$ follow this curve very closely which is consistent with our correlation dimension analysis above. This observation further reinforces our conjecture that ordinal-network-based Markovian-walk surrogates (for larger $m$ values) preserve density characteristics of the state-space attractor and a high degree of self-similarity.
We postulate that surrogates lose some degree of selfsimilarity in smaller scales in comparison to the original data. This effect is more pronounced for surrogates produced by ordinal networks of smaller $m$. In other words, a sufficiently high $m$ is required in order to uncover the finer details of the state-space attractor. However, large-scale characteristics and certain complexity properties are preserved by this Markovian model whereby successive states are allowed a maximal overlap.

\section{CONCLUSION}

In this study, we have constructed ordinal networks using discrete sample time series from a continuous chaotic Lorenz system and then used random walks on the network models to regenerate surrogate time series data. We considered two variants of this process: random walks constrained to allowable ordinal transitions on networks constructed with time lag $\tau>1$ and Markovian random walks on networks constructed with $\tau=1$. Respective investigations of both cases demonstrate that ordinal network models can generate random walk stochastic time series that approximate the dynamical properties of the original time series for sufficiently large embedding dimension $m$.

For constrained random walks on networks constructed with $\tau>1$, we find that the order recurrence plots for both original and random walk symbolic dynamics comprise similar structural patterns-predominately characterised by short diagonal lines of various lengths as expected given the quasi-periodic dynamics. However, the order recurrence plot for the constrained random walk appears to exhibit more regularity and shorter epochs of these diagonal structures. Furthermore, while the recurrence quantification analysis measures of vertical line structures are relatively consistent between the original and random walks, measures of diagonal line structures diverge as the size of the ordinal network approaches the length of available data.

In the case of Markovian random walks on networks constructed with $\tau=1$, we computed the correlation dimension of the original time series against the network generated surrogates and showed that the surrogate time series approaches a fractal set with increasing $m$. Our results also indicate relative consistency between the original and surrogate time series for the first minimum of the time delayed mutual information given sufficiently large $m$.

We also investigated the average separation of nearby trajectories in network generated surrogate time series for constrained random walks and Markovian random walks. Our results indicate that the dynamics of the surrogates approximate the deterministic exponential divergence characteristic of the original time series, and hence, the network model preserves some information pertaining to the largest Lyapunov exponent. However, the observable scaling region for the surrogates is consistently shorter than for the original time series.

The overarching finding from our investigations is that ordinal networks constructed from univariate time series data constitute stochastic models which approximate important dynamical properties of the original systems. These 
properties include the qualitative appearance of the time series, recurrence properties, and invariant properties. For interested readers, we reiterate that selected results for additional systems are collated as supplementary available online.

\section{SUPPLEMENTARY MATERIAL}

See supplementary material for a selection of additional results for time series generated by other archetypal chaotic systems including the Rössler system, the Chua system, and the four dimensional hyper-chaotic Rössler system.

\section{ACKNOWLEDGMENTS}

M.S. was supported by the Australian Research Council Discovery Project DP 140100203.

${ }^{1}$ J. Zhang and M. Small, Phys. Rev. Lett. 96, 238701 (2006).

${ }^{2}$ R. V. Donner, M. Small, J. F. Donges, N. Marwan, Y. Zou, R. Xiang, and J. Kurths, Int. J. Bifurcation Chaos Appl. Sci. Eng. 21, 1019 (2011)

${ }^{3}$ M. Thiel, M. C. Romano, and J. Kurths, Phys. Lett. A 330, 343 (2004).

${ }^{4}$ Y. Hirata, S. Horai, and K. Aihara, Eur. Phys. J. Spec. Top. 164, 13 (2008).

${ }^{5}$ A. S. L. O. Campanharo, M. I. Sirer, R. D. Malmgren, F. M. Ramos, and L. A. N. Amaral, PLoS ONE 6, e23378 (2011).
${ }^{6}$ L. Hou, M. Small, and S. Lao, Entropy 17, 6433 (2015).

${ }^{7}$ Y. Hirata, A. Oda, K. Ohta, and K. Aihara, Sci. Rep. 6, 34982 (2016).

${ }^{8}$ M. McCullough, M. Small, T. Stemler, and H. H. C. Iu, Chaos 25, 053101 (2015).

${ }^{9}$ C. Bandt and B. Pompe, Phys. Rev. Lett. 88, 174102 (2002).

${ }^{10}$ J. M. Amigó, K. Keller, and V. A. Unakafova, Philos. Trans. R. Soc. London A 373 (2014).

${ }^{11}$ K. Keller and M. Sinn, Physica D 239, 997 (2010).

${ }^{12}$ J. Amigó and K. Keller, Eur. Phys. J. Spec. Top. 222, 263 (2013).

${ }^{13}$ A. Groth, Phys. Rev. E 72, 046220 (2005).

${ }^{14}$ N. Marwan, M. C. Romano, M. Thiel, and J. Kurths, Phys. Rep. 438, 237 (2007).

${ }^{15}$ E. N. Lorenz, J. Atmos. Sci. 20, 130 (1963).

${ }^{16}$ Y. Cao, W. Tung, J. B. Gao, V. A. Protopopescu, and L. M. Hively, Phys. Rev. E 70, 046217 (2004).

${ }^{17}$ L. Zunino, M. C. Soriano, I. Fischer, O. A. Rosso, and C. R. Mirasso, Phys. Rev. E 82, 046212 (2010).

${ }^{18}$ This can only occur when the final symbol or sequence of symbols in the symbolic dynamics has not occurred at any prior time in dynamics, which is very unlikely in practice as long as the size of the network is much greater than the length of the time series.

${ }^{19}$ T. Schreiber and A. Schmitz, Physica D 142, 346 (2000).

${ }^{20}$ J.-P. Eckmann, S. O. Kamphorst, and D. Ruelle, EPL (Europhysics Letters) 4, 973 (1987).

${ }^{21}$ R. Hegger, H. Kantz, and T. Schreiber, Chaos 9, 413 (1999).

${ }^{22}$ M. T. Rosenstein, J. J. Collins, and C. J. D. Luca, Physica D 65, 117 (1993).

${ }^{23}$ H. Kantz, Phys. Lett. A 185, 77 (1994).

${ }^{24}$ P. Grassberger and I. Procaccia, Phys. Rev. Lett. 50, 346 (1983). 